\title{
An Automated Composite Table Algorithm Considering Zero Liquid Discharge Possibility in Water Regeneration-recycle Network
}

Reza Parand ${ }^{a, b,}{ }^{*}$, Hong Mei Yao ${ }^{a}$, Dominic C.Y. Foo ${ }^{c}$, Moses O. Tadéa

a Department of Chemical Engineering, Curtin University, GPO Box U1987, Perth, WA 6845, Australia;

${ }^{b}$ Australasian Joint Research Centre for Building Information Modelling, School of Built Environment, Curtin University, GPO Box U1987, Perth, WA 6845, Australia;

${ }^{\mathrm{C}}$ Centre of Excellence for Green Technologies/Department of Chemical and Environmental Engineering, University of Nottingham Malaysia, Broga Road, 43500 Semenyih, Selangor, Malaysia;

\section{Abstract}

In this study, a novel Automated Composite Table Algorithm (ACTA) is developed for targeting the water regeneration-recycle network of single contaminant problem. The ACTA is based on Pinch Analysis, but is automated by taking into consideration of the possibility of zero liquid discharge (ZLD) for the water network. In the existing literature, the targeting procedure for ZLD network is based on the graphical tool of Limiting Composite Curve (LCC). However, identification of key parameters (i.e. freshwater, wastewater, regenerated water flowrates, along with pre-regeneration concentrations) is very tedious for highly integrated water network system. The magnification around the turning point of LCC is required to identify the correct pinch points; and targeting procedure is done iteratively until the reliable network targets can be determined. These limitations are now overcome by the ACTA, which is an improved version of Composite Table Algorithm that is capable of identifying key parameters algebraically for a given post-regeneration concentration. The newly developed ACTA is capable of handling a wide range of problems including ZLD and non-ZLD network, for both fixed load and fixed flowrate problems.

Keywords: Process Integration, Pinch Analysis, water minimisation, targeting, optimization.

\section{Introduction}

Human activity is arguably the biggest contributor for the imbalance natural ecological systems. In that regard, climate change is probably the most important environmental issue faced by the modern world today (Steffen et al., 2015). Hence, sustainable development has been a major topic of interest in the past few decades. Within the chemical engineering domain, various policy papers have also been made to promote sustainable development, especially for the process industry. For instance, the Institute of Chemical Engineers (IChemE) has published a technical roadmap to handle four important challenging areas (termed as vista) of the modern society, which include water, energy, food and wellbeing (IChemE, 2014). For the water vista, reuse and recycle have been recognised as an important mean for immediate action, in which cost effective techniques should be developed to enable the effective recovery of water resources in the process plants (IChemE, 2014).

Within the research area of process system engineering, some systematic works on water recovery has been reported as early as 1980s (Takama et al., 1980). However, the research area did not get much attention until

\footnotetext{
${ }^{*}$ Corresponding author. Telephone: +61 8 9266564. E-mails: reza.parand@curtin.edu.au (R.Parand); H.Yao@curtin.edu.au (H.M.Yao); Dominic.Foo@nottingham.edu.my (D.C.Y.Foo); M.O.Tade@curtin.edu.au (M.O.Tadé)
} 
mid-90s, where a special case was developed within the Process Integration (PI) community, focusing on water minimisation using Pinch Analysis technique (or Water Pinch Analysis in short). In the seminal work of Wang and Smith (1994), water demand reduction can be achieved for the process plants through process changes, water reuse/recycle and regeneration. The authors also proposed the graphical targeting tool called the limiting composite curve (LCC) to identify the minimum flowrate targets for the various water recovery strategies. Since then, many different targeting techniques were developed. Some recent developments, methodologies, and successful industrial applications of PI are now well documented in a handbook (Klemeš, 2013), review paper (Foo, 2009) and even textbooks for process integration (Foo, 2012) and for genetic process design (Smith, 2016). Specifically, some recent developments for water pinch related studies can be found in Klemeš et al. (2014).

Among the various water recovery strategies, direct reuse/recycle is often being taken as the first priority, as it involves minimum expenditure. Once the maximum potential for recovery is exhausted, one then switches to perform water regeneration, where water purification unit(s) is used to upgrade quality of water sources for further reuse/recycle. As mentioned earlier, the targeting for water regeneration-recycle network was first reported by Wang and Smith (1994), however was dedicated for the fixed load (FL) problems, where waterusing processes were assumed as mass transfer operations. The same group of authors (Kuo and Smith, 1998) later extended the LCC for targeting for water regeneration network, however was also limited to FL problems. The review of Foo (2009) identified that most works reported in the $21^{\text {st }}$ century were dedicated to the fixed flowrate (FF) problem. The latter is considered more generic as it includes water-using processes other than those of mass transfer-based. The reported works include those for data extraction (Foo et al., 2006), flowrate targeting (e.g. Saw et al, 2009) and network design (e.g. Aly et al., 2005). The first guideline for regeneration placement in the FF problems was proposed by Hallale (2002). It was suggested that in order to reduce the overall freshwater and wastewater flowrates, the regeneration unit should be placed across the reuse/recycle pinch concentration (Hallale, 2002). In this way, wastewater will be collected from the region with a surplus of water, purified and be fed to the region with water deficit. Following this guideline, Material Recovery Pinch Diagram (El-Halwagi, 2006) and Water Cascade Analysis (Manan et al., 2004) have been used to determine the various flowrate targets for a water regeneration network. Note however that all these methods cannot guarantee the minimum freshwater, wastewater, and regenerated flowrates to be achieved simultaneously. This limitation was then rectified by $\mathrm{Ng}$ et al. (2007) through an algebraic procedure. However, since the procedure was developed on the basis of flowrate reallocation of Kuo and Smith (1998), which is iterative in nature and is tedious during implementation. Bandyopadhyay et al. (2006) proposed Source Composite Curve for targeting the water regeneration-recycle network. The Improved Problem Table (IPT) was developed by Deng and Feng (2011) for targeting multiple water resources with inclusion of regeneration unit. Deng et al. (2016) recently extended the IPT for targeting water regeneration network with multiple partitioning interception units.

Another important targeting tool for water regeneration network was proposed by Agrawal and Shenoy (2006), which made use of the composite table algorithm (CTA) to identify other key parameters for a given 
post regeneration concentration $\left(C_{0}\right)$. The CTA first generates the data in tabular form, and displays the targeting results using the LCC. However, the main assumption of this work is that, the reuse/recycle pinch is taken as pinch point in water regeneration system. All key steps to determine the key parameters are developed based on this assumption. Note however that the pinch point(s) in a water regeneration-recycle system can vary with different $C_{0}$ values (to be elaborated later). Thus, the targeted results obtained by original CTA may not be reliable for some cases. Parand et al. (2013a) demonstrated that the CTA is capable of handling various problems (i.e. FL, FF, and their hybrid, etc.) in water reuse/recycle network. Also, Parand et al. (2014) proposed the use of Composite Matrix Analysis to analyse the interactions among key parameters in a total regeneration water network.

Among the various reported works, one of the special cases is called the threshold problem, in which water network does not feature fresh water intake and/or wastewater discharge; the latter is also termed the zero liquid discharge (ZLD) situation. Foo (2008) first reported the targeting for several types of threshold problems for reuse/recycle systems. Parand et al. (2013b) later proposed the harvesting of impure external water sources for saving freshwater supply in the ZLD problems. In addition, ZLD may also achieve in a water regeneration network when appropriate regeneration unit(s) is used. For such cases, the freshwater should supplement the amount of water loss. Deng et al. (2008) first utilised the LCC to identify the key parameters for a water regeneration network to achieve ZLD. Note however that the turning points of LCC may not be vividly recognisable for highly integrated water network. Thus, identifying the key parameters using the LCC may be a cumbersome task, as magnification around the turning points of LCC is required to find the correct pinch points. Also the targeting procedure should be done iteratively until the reliable targets are found. In this study, a novel methodology named the Automated Composite Table Algorithm (ACTA) is developed. The ACTA is based on the algebraic targeting procedure that were originally proposed by Feng et al. (2007) and Deng et al. (2008), however is automated with improved procedure for both data generation and target identification steps. The ACTA is a rigorous targeting methodology for water regeneration-recycle network which can handle a wide range of problems, including ZLD and non-ZLD water networks, as well as FL and FF problems. The paper is structured as follows. In the following section, a formal problem statement is first presented. Next, the targeting concept of a regeneration-recycle system is illustrated using the LCC. The newly proposed ACTA procedure is then illustrated using a FF problem in Example 1. Example 2 on FL problem is next demonstrated to show the applicability of the ACTA, before the final conclusions are drawn.

\section{Problem statement}

Consider a water network that consists of the following units:

- Processes that demand water, designated as process $\operatorname{sinks}$ or $\operatorname{SKj}(j=1,2, \ldots \mathrm{m})$. Every sink requires a highest concentration limit $\left(C_{\mathrm{SKj}}^{\max }\right)$, i.e. $C_{\mathrm{SKj}} \leq C_{\mathrm{skj}}^{\max }$ 
- Processes that produce water, in which may be sent for reuse or recycle to the process sinks, designated as process sources, or SRi $(i=1,2, \ldots, \mathrm{n})$. Each source has a fixed flowrate $\left(F_{\mathrm{SRi}}\right)$, and an impurity concentration $\left(C_{S R i}\right)$.

- Water regeneration unit of known performance i.e. fixed post-regeneration $\left(C_{0}\right)$, that may be use to purify water sources before they are reused/recycled to the process sinks. Water sources enter regeneration unit at pre-regeneration concentration $\left(C_{\text {reg }}\right)$. The flowrate loss for the regeneration unit is assumed to be negligible. This is termed as the single pass regeneration unit (Foo, 2012).

- When the process sinks cannot be satisfied by the process sources, either due to quality (contaminant mass load) and quantity (flowrate) constraints, an outsourced freshwater (regarded as an external source) with flowrate of $F_{\mathrm{fw}}$ and contaminant concentration of $C_{\mathrm{fw}}$ is purchased to supplement the flowrate requirement of the sinks. Unused water from process sources (if any) will be disposed as waste stream (with concentration of $C_{\mathrm{ww}}$ and flowrate of $F_{\mathrm{ww}}$ ).

Superstructure presentation of the problem is depicted in Figure 1. The objective of the problem is to determine the various flowrate targets i.e. $F_{\mathrm{fw}}, F_{\mathrm{reg}}, F_{\mathrm{ww}}$ along with the other important parameters (e.g. $C_{\mathrm{reg}}$ and $C_{w w}$ ) that are needed to design a water regeneration-recycle network.

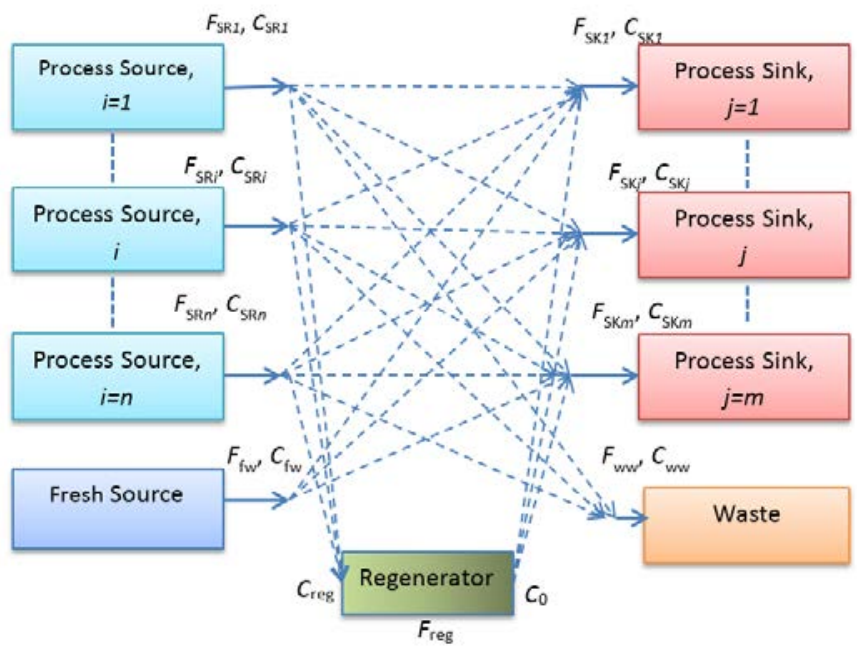

Figure 1. Superstructure presentation of water regeneration network (Parand et al., 2014)

\section{Targeting regeneration-recycle system with LCC}

In order to better illustrate the newly proposed ACTA, the characteristic for targeting a water regenerationrecycle network is first explained using the LCC in Figure 2 (Feng et al., 2007). 


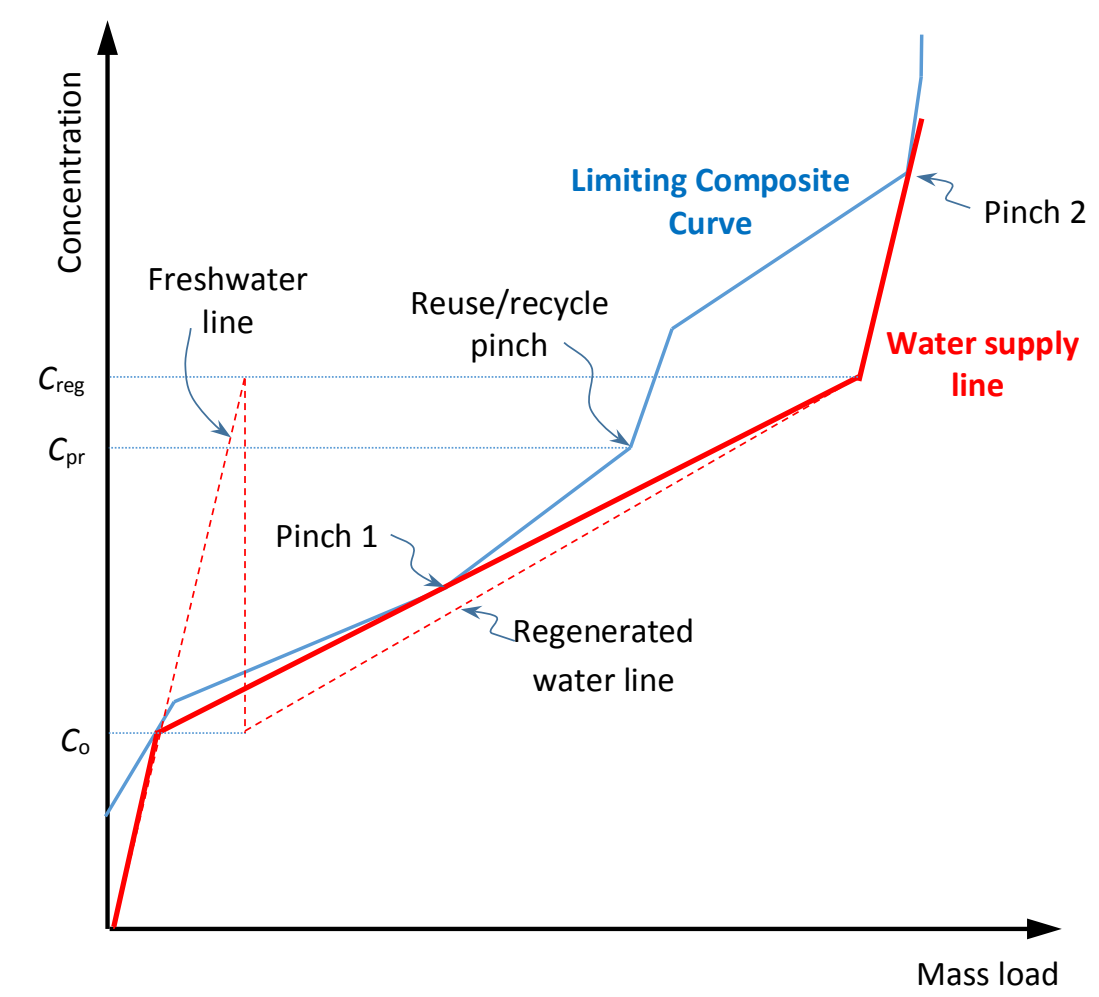

1

2 Figure 2. LCC and water supply line for targeting water regeneration-recycle system

3 The water supply line (WSL) in Figure 2 (red solid line) represents the composite curve for the freshwater line

4 (indicated by dotted line between origin and $C_{\text {reg }}$ ) and regenerated water line (dotted line between $C_{0}$ and $C_{\text {reg, }}$

5 with lower slope). The inverse slope of these segments identifies the optimum freshwater $\left(F_{\mathrm{fw}}\right)$ regenerated

6 water flowrates $\left(F_{\text {reg }}\right)$ for the system. With the combination of the freshwater and regenerated water lines,

7 the WSL starts from the origin and turns its direction at post- $\left(C_{0}\right)$ and pre-regeneration $\left(C_{\text {reg }}\right)$ concentrations,

8 while touches the LCC at two pinch points, i.e. Pinch 1 and Pinch 2. These latter are considered as regeneration pinch points of the water regeneration-recycle system. Note that the existence of two pinch points (e.g. Pinch 1 and Pinch 2 in Figure 2 ) is very common in a water regeneration-recycle system (Feng et al., 2007) . However, the locations of these pinch point are dependent on the given $C_{0}$ value and the turning points of LCC, as these points dictate the shape of the WSL. Note also that a small portion of the freshwater line that forms the WSL (see Figure 2) actually moves along the LCC with the change of $C_{0}$ (Feng et al., 2007).

The ZLD network is a special case of water regeneration-recycle system. For some cases, utilising the original LCC targeting procedure (Feng et al., 2007) to determine the water flowrate targets may lead to infeasible result (i.e. negative wastewater flowrate). This phenomenon happens for a confined range of $C_{0}$. Thus, the LCC needs to be updated with new boundary in order to rectify the infeasibility issue. As such, the limiting data need to be modified to reflect this update. The ACTA developed in this study is capable of detecting the possibility of ZLD, which then updates the limiting data in order to determine the rigorous water network targets. The ACTA has been implemented using MATLAB. The implementation is explained explicitly through a literature example in the following sections. 


\section{The new ACTA procedure (Example 1)}

Example 1 is taken from Polley and Polley (2000) to illustrate the newly proposed methodology. The limiting data is given in Table 1. The water network for example 1 consists of four FF water-using operations. The network requires $300 \mathrm{t} / \mathrm{h}$ of freshwater (the sum of flowrates for all process sinks) and generates $280 \mathrm{t} / \mathrm{h}$ of wastewater (the sum of the flowrates for process sources), when no water recovery scheme is considered. Also, this network encounters $20 \mathrm{t} / \mathrm{h}$ of water loss (difference between freshwater and wastewater flowrates) and a regeneration unit with $C_{0}=20 \mathrm{ppm}$ is utilized for this network.

The first step is to identify the flowrate targets and the pinch location for water reuse/recycle network.

Table 1. Limiting data for example 1 (Polley and Polley, 2000)

\begin{tabular}{llllll}
\hline SKj & $F_{\text {SKj }}(\mathrm{t} / \mathrm{h})$ & $C_{\text {SKj }}(\mathrm{ppm})$ & $\mathrm{SRi}$ & $F_{\mathrm{SRi}}(\mathrm{t} / \mathrm{h})$ & $C_{\mathrm{SRi}}(\mathrm{ppm})$ \\
\hline 1 & 50 & 20 & 1 & 50 & 50 \\
2 & 100 & 50 & 2 & 100 & 100 \\
3 & 80 & 100 & 3 & 70 & 150 \\
4 & 70 & 200 & 4 & 60 & 250 \\
\hline
\end{tabular}

\section{Step 1. Flowrate targets and pinch point identification for reuse/recycle scheme}

The CTA procedure (Agrawal and Shenoy, 2006) is employed to identify the flowrate targets for a direct reuse/recycle network (Table 2). The detailed procedure is outlined as follows:

- To form concentration levels $\left(C_{\mathrm{k}}\right)$ : All limiting concentrations $\left(C_{\mathrm{SK} j}, C_{\mathrm{SR} i}\right)$ including a highest arbitrary value (e.g. 300 ppm) are grouped and arranged in ascending order.

- To determine the interval flowrates $\left(\operatorname{Net} F_{k}\right)$ : For each concentration interval $\left(C_{k}, C_{k+1}\right)$, the total flowrate of process sources is subtracted from that of process sinks. To conveniently determine the stream population in each interval, the streams are shown as vertical arrows in SK1-SK4 and SR1-SR4 columns. The numbers in stream columns indicate the flowrate for each stream. Each stream starts from its concentration and ends at the arbitrary value. In this way, the Net $F_{\mathrm{k}}$ can be easily determined for each concentration interval.

- To identify the interval impurity loads $\left(\Delta m_{\mathrm{k}}\right)$ : These values are calculated by multiplying the interval flowrate (Net $\left.F_{k}\right)$ with the difference of concentration levels $\left(C_{\mathrm{k}+1}-C_{\mathrm{k}}\right)$.

- To identify the cumulative load (Cum. $\Delta m_{\mathrm{k}}$ ): By assuming the zero impurity load as the first entry, the Cum. $\Delta m_{\mathrm{k}}$ is calculated by cascading down the interval impurity load $\left(\Delta m_{\mathrm{k}}\right)$.

- To calculate the interval freshwater flowrate $\left(F_{\mathrm{fw}, \mathrm{k}}\right)$ : The interval freshwater flowrate is calculated via Eq.1. Note that for this study, pure freshwater is assumed (i.e. $C_{\mathrm{fw}}=0 \mathrm{ppm}$ ).

$F_{f w, k}=\frac{C u m \Delta m_{k}}{C_{k}-C_{f w}}$ 


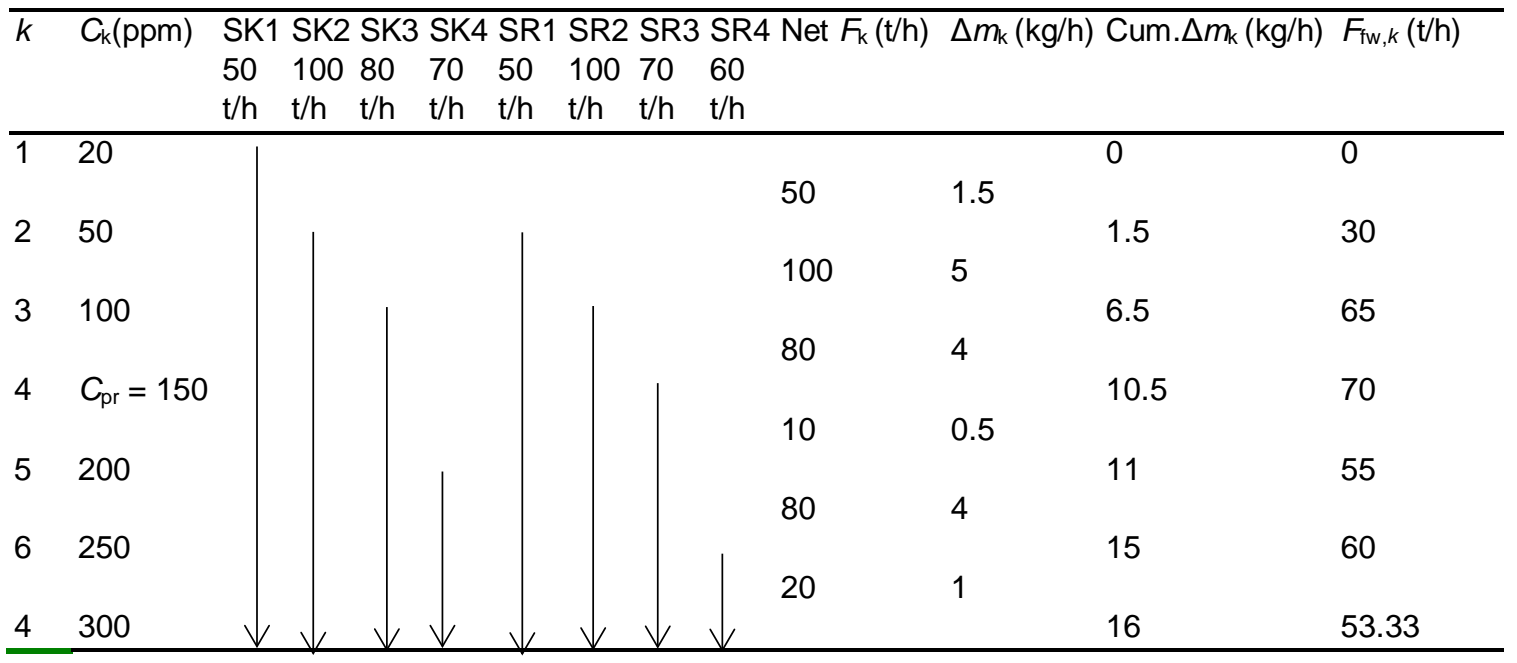

2

The largest value among the entries in the last column of Table 2 identifies the optimum freshwater flowrate for reuse/recycle network, i.e. $F_{\mathrm{fw}}=70 \mathrm{t} / \mathrm{h}$, with a pinch concentration $\left(C_{\mathrm{pr}}\right)$ located at $150 \mathrm{ppm}$ (corresponds to the level where minimum freshwater flowrate is found). Due to $20 \mathrm{t} / \mathrm{h}$ of water loss, the wastewater flowrate is then calculated as $50 \mathrm{t} / \mathrm{h}(=70-20 \mathrm{t} / \mathrm{h})$. As explained, this network has the potential for ZLD due to water loss; this will be evaluated in the following step.

\section{Step 2. Checking the possibility of ZLD}

To better illustrate this step, the LCC for Example 1 is shown in Figure 3, plotted using concentration level (column 2) against cumulative load values (column 5) of Table 2. The last segment of the LCC (line $A^{\prime} B^{\prime}$ ) represents the amount of total water loss of this network (with its inverse slope indicating the flowrate). Hence, the amount of freshwater needed by this network has to be able to supplement this water loss. In other words, the freshwater flowrate has to be at least equal to (or bigger than) the water loss of the network, which will be determined by the ACTA. The ZLD network is then attainable with appropriate use of regeneration unit, in which process sources are partially purified for further reuse/recycle to the process sinks. Graphically, line $A B$ is drawn from the origin in parallel with the last segment of LCC (i.e. line $\left.A^{\prime} B^{\prime}\right)$. As such, line $A B$ will have an inverse slope that corresponds to the flowrate which equals to the amount of water loss of the water network. Note that the freshwater line can overlap with line $A B$, depending on the given $C_{0}$ value. Thus, the freshwater line introduced this way can supply the amount of water loss experienced by the water network. Note however that line AB should intersect with the LCC below the reuse/recycle pinch point (i.e. $150 \mathrm{ppm}$ for Example 1). The main reason is that, freshwater should only be used to supply the process sinks in the high quality region (with the concentration lower than reuse/recycle pinch point). The intersection point between line $A B$ and the $L C C$ is termed as the maximum post-regeneration concentration for $Z L D\left(C_{o, Z L D}^{\max }\right)$. For Example 1, the $C_{o, Z L D}^{\max }$ value is identified at $33.33 \mathrm{ppm}$ (refer to the appendix for detailed numerical 

network.

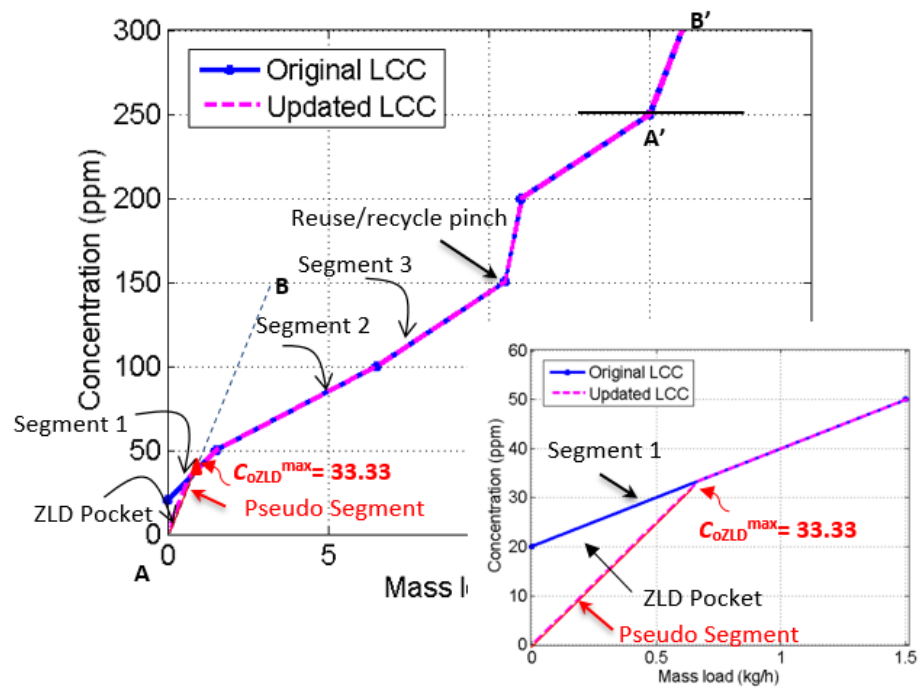

3

4

5

6

Figure 3. LCC to check the possibility of ZLD for Example 1

A pseudo segment is introduced along line AB in Figure 3, which starts at the origin and intercepts with the LCC at $C_{o, Z L D}^{\max }$. The area enclosed among the pseudo segment, LCC, and Y-axis is termed as the ZLD pocket (see the enlarged version of Figure 3 for clearer illustration). Clearly, if the freshwater line appears within this region (with slope higher than that of the pseudo segment), the targeted value for freshwater flowrate will be lower than the amount of water loss, which in turn leads to negative waste water flowrate (i.e. an infeasible result). Hence, the upper line of ZLD pocket needs to be removed, so to prevent the possibility of infeasible flowrate targets.

Following the removal of the upper line of ZLD pocket, the limiting data for the water network needs to be updated (to be discussed in step 3). As a result, an updated LCC will be constructed. As demonstrated in Figure 3 , the updated LCC starts from origin, continues on the pseudo segment until it reaches the $C_{o, Z L D}^{\max }$, and finally merges with the original LCC thereafter. The detailed algebraic procedure of this step to be implemented with MATLAB is found in the appendix.

\section{Step 3. Update of limiting data for ZLD pocket removal}

In order to plot the updated LCC, the limiting data need to be updated, and is given in Table 3 . The modified values (as compared with original ones in Table 2 ) are shown in bold. The detailed procedure to generate the updated limiting data is discussed as follows: 
Table 3. Updated limiting data with the removal of the upper line of ZLD pocket

\begin{tabular}{llllll}
\hline SKj & $F_{\text {SKj }}(\mathrm{t} / \mathrm{h})$ & $C_{\text {SKj }}(\mathrm{ppm})$ & $\mathrm{SRi}$ & $F_{\mathrm{SR} i}(\mathrm{t} / \mathrm{h})$ & $C_{\mathrm{SRi}}(\mathrm{ppm})$ \\
\hline Pseudo & $\mathbf{2 0}$ & $\mathbf{0}$ & Pseudo & $\mathbf{2 0}$ & $\mathbf{3 3 . 3 3}$ \\
1 & 50 & $\mathbf{3 3 . 3 3}$ & 1 & 50 & 50 \\
2 & 100 & 50 & 2 & 100 & 100 \\
3 & 80 & 100 & 3 & 70 & 150 \\
4 & 70 & 200 & 4 & 60 & 250 \\
\hline
\end{tabular}

3

4

5

6

\section{Modification of limiting concentration data}

In order to remove the ZLD pocket, all turning points of the LCC below the $C_{o, Z L D}^{\max }$ should be removed from analysis. In doing so, all concentration for sinks $\left(C_{\mathrm{SK} j}\right)$ and sources $\left(C_{\mathrm{SR} i}\right)$ lower than $C_{0, Z L D}^{\max }$ should be replaced by $C_{o, Z L D}^{\max }$; the latter has been identified as $33.33 \mathrm{ppm}$ in step 2. This corresponds to SK1 (20 ppm, see Table 1 ) for Example 1, with its concentration now is changed to 33.33 ppm (Table 3).

\section{Inclusion of pseudo sink and pseudo source}

Once the turning point(s) of LCC lie below the $C_{o, Z L D}^{\max }$ is removed, the pseudo segment (shown in Figure 3 ) is added for analysis. To do so, a pair of pseudo sink and pseudo source is introduced for the limiting data, with their flowrates correspond to the amount of water loss, i.e. $20 \mathrm{t} / \mathrm{h}$ for Example 1. The pseudo sink gets the concentration of $0 \mathrm{ppm}$, representing the starting point of the pseudo segment at the origin. On the other hand, the pseudo source will have its concentration equal to $C_{o, Z L D}^{\max }$, i.e. $33.33 \mathrm{ppm}$ in this case, representing the ending point of the pseudo segment. In other words, the pseudo sink and source are the algebraic representation for the pseudo segment added to form the updated LCC (in Figure 3). Once the ZLD pocket is removed, the procedure moves forward to step 4 for identification of the key parameters.

\section{Step 4. Identification of freshwater $\left(F_{\mathrm{fw}}\right)$ and regenerated water flowrates $\left(\boldsymbol{F}_{\mathrm{reg}}\right)$, and pre-regeneration concentration $\left(C_{\mathrm{reg}}\right)$ for the given $\mathrm{C}_{\mathrm{o}}$}

In this step, the important key parameters in water regeneration-recycle network (with $C_{\mathrm{o}}=20 \mathrm{ppm}$ ) are identified by using the updated limiting data. To do that, the original CTA (Agrawal and Shenoy, 2006) is improved in this work. The result of the improved CTA is shown in Table 4. Note that the first five steps are the same as original CTA (Agrawal and Shenoy, 2006) outlined in step 1. The stream population is eliminated in this table for the purpose of brevity. Note also that during the formation of the concentration levels $\left(C_{\mathrm{k}}\right.$, in step 1 ), the $C_{o}$ should also be included. Among the various $F_{f w, k}$, values in column 6 (identified via Eq. 1 in step 1), the entry at the $C_{0}$ level $(20 \mathrm{ppm})$ is identified as the optimum freshwater flowrate for the water regenerationrecycle network (i.e. $F_{\mathrm{fw}}=20 \mathrm{t} / \mathrm{h}$ ).

Two more steps are added for the improved CTA (adopted from the work of Feng et al., 2007) as follows: 
$4 \quad F_{r e g, k}=\frac{C u m \Delta m_{k}-F_{f w} \times C_{k}}{C_{k}-C_{0}}, \forall k \rightarrow C_{o}<C_{k} \leq C_{p r}$ i.e. $20 \mathrm{ppm} \leq C_{\mathrm{k}} \leq 150 \mathrm{ppm}$. concentration of 150 ppm ( $C_{\text {Pinch } 1}=150$ ppm; see Figure 4$)$.

$$
C_{r e g, k}=\frac{C u m . \Delta m_{k}-F_{f w} \times C_{k}+F_{r e g} \times C_{o}}{F_{r e g}}, \forall k \rightarrow C_{p r} \leq C_{k}
$$

- $\quad$ To identify the interval regenerated water flowrate $\left(F_{\text {reg,k }}\right)$ : These flowrates are calculated using Eq. 2 (Feng et al., 2007). Note that Eq. 2 considers the concentration levels $\left(C_{k}\right)$ which lie between $C_{o}$ and $C_{p r}$,

The largest value among all entries in column 7 identify the optimum regenerated water flowrate (i.e. $F_{\text {reg }}=$ $57.69 \mathrm{t} / \mathrm{h})$. The $C_{\mathrm{k}}$ where $F_{\text {reg }}$ is located hence identifies the first regeneration pinch for this network, with

- To identify the associated pre-regeneration concentration levels $\left(C_{\mathrm{reg}, \mathrm{k}}\right)$ : These concentration levels are determined using Eq. 3 (Feng et al., 2007), which considers $C_{\mathrm{k}} \mathrm{S}$ which stay between $C_{\mathrm{pr}}$ and the largest arbitrary concentration level, i.e. $150 \mathrm{ppm} \leq C_{\mathrm{k}} \leq 300 \mathrm{ppm}$.

The largest value among all entries in column 8 of Table 4 next identifies the optimum pre-regeneration concentration, i.e. $C_{\text {reg }}=193.33 \mathrm{ppm}$. In addition, the $C_{\mathrm{k}}$ value at the same level where $C_{\text {reg }}$ is located identifies the second regeneration pinch, with concentration of $250 \mathrm{ppm}\left(C_{\text {Pinch 2, }}\right.$, see Figure 4).

Table 4. Determination of $F_{\mathrm{fw}}, F_{\mathrm{reg}}$, and $C_{\mathrm{reg}}$ with improved CTA for $C_{\mathrm{o}}=20 \mathrm{ppm}$ (Example 1)

\begin{tabular}{|c|c|c|c|c|c|c|c|}
\hline$k$ & $C_{k}(p p m)$ & Net. $F_{\mathrm{k}}(\mathrm{t} / \mathrm{h})$ & $\Delta m_{\mathrm{k}}(\mathrm{kg} / \mathrm{h})$ & Cum. $\Delta m_{\mathrm{k}}(\mathrm{kg} / \mathrm{h})$ & $F_{\mathrm{fw}, k}(\mathrm{t} / \mathrm{h})$ & $F_{\text {reg,k }}(\mathrm{t} / \mathrm{h})$ & $C_{\text {reg, } \mathrm{k}}(\mathrm{ppm})$ \\
\hline \multirow[t]{2}{*}{1} & 0 & & & 0 & 0 & & \\
\hline & & 20 & 0.4 & & & & \\
\hline \multirow[t]{2}{*}{2} & $C_{0}=20$ & & & 0.4 & $F_{\mathrm{fw}}=20$ & & \\
\hline & & 20 & 0.27 & & & & \\
\hline \multirow[t]{2}{*}{3} & 33.33 & & & 0.67 & 20 & 0 & \\
\hline & & 50 & 0.83 & & & & \\
\hline \multirow[t]{2}{*}{4} & 50 & & & 1.5 & 30 & 16.67 & \\
\hline & & 100 & 5 & & & & \\
\hline \multirow[t]{2}{*}{5} & 100 & & & 6.5 & 65 & 56.25 & \\
\hline & & 80 & 4 & & & & \\
\hline \multirow[t]{2}{*}{6} & $C_{\text {pinch } 1}=150$ & & & 10.5 & 70 & $F_{\text {reg }}=57.69$ & 150 \\
\hline & & 10 & 0.5 & & & & \\
\hline \multirow[t]{2}{*}{7} & 200 & & & 11 & 55 & & 141.33 \\
\hline & & 80 & 4 & & & & \\
\hline \multirow[t]{2}{*}{8} & $C_{\text {pinch 2 }}=250$ & & & 15 & 60 & & $C_{\text {reg }}=193.33$ \\
\hline & & 20 & 1 & & & & \\
\hline 9 & 300 & & & 16 & 53.33 & & 193.33 \\
\hline
\end{tabular}

17 Step 5. Determination of wastewater flowrate $\left(F_{w w}\right)$ and concentration $\left(C_{w w}\right)$

18 Two other important parameters for a water regeneration-recycle network are to be determined in this step,

19 i.e. wastewater flowrate $\left(F_{w w}\right)$ and its concentration $\left(C_{w w}\right)$. 
8

9

The $F_{w w}$ target can be calculated using Eq. 4 (Agrawal and Shenoy, 2006). The right hand side terms of Eq. 4 determines the amount of water loss/gain for a particular network. The water network in Example 1 encounters water loss of $20 \mathrm{t} / \mathrm{h}$. With its $F_{\mathrm{fw}}$ identified at $20 \mathrm{t} / \mathrm{h}$ in step 4, the $F_{\mathrm{ww}}$ is easily determined as $0 \mathrm{t} / \mathrm{h}$.

This verifies that a ZLD network is achievable for this example, when a regeneration unit of $C_{0}=20 \mathrm{ppm}$ is used. In other words, contaminants in the process sources are partially removed by the regeneration unit before they are recycled to the sinks. Note that $C_{o, Z L D}^{\max }$ is identified at $33.33 \mathrm{ppm}$. Thus, the ZLD network can be achieved for $C_{0}$ s range of $0-33.33 \mathrm{ppm}$.

$F_{f w}-F_{w w}=\sum_{i} F_{S K i}-\sum_{j} F_{S R j}$

$F_{f w} \times C_{f w}-F_{w w} \times C_{w w}-F_{r e g}\left(C_{r e g}-C_{o}\right)=\sum_{i} F_{S K i} C_{S K i}-\sum_{i} F_{S R j} C_{S R j}$

The $C_{\text {ww }}$ can be readily calculated via Eq. 5. For Example 1, since the network generates no wastewater, hence this step is omitted.

By this end, the optimum rigorous values of all key parameters in a regeneration-recycle network $\left(F_{\mathrm{fw}}, F_{\mathrm{reg}}, C_{\mathrm{reg}}\right.$, $F_{\mathrm{ww}}$, and $C_{\mathrm{ww}}$ ) have been identified for $C_{0}=20 \mathrm{ppm}$. The values obtained through ACTA are completely in compliance with those reported by Agrawal and Shenoy (2006).

These targeting results are also displayed graphically on the LCC in Figure 4. The WSL (dotted line) stays entirely below and touches the LCC (rigid line) at the two regeneration pinch points. This graphical presentation verifies the accuracy of the ACTA for targeting a water regeneration-recycle network

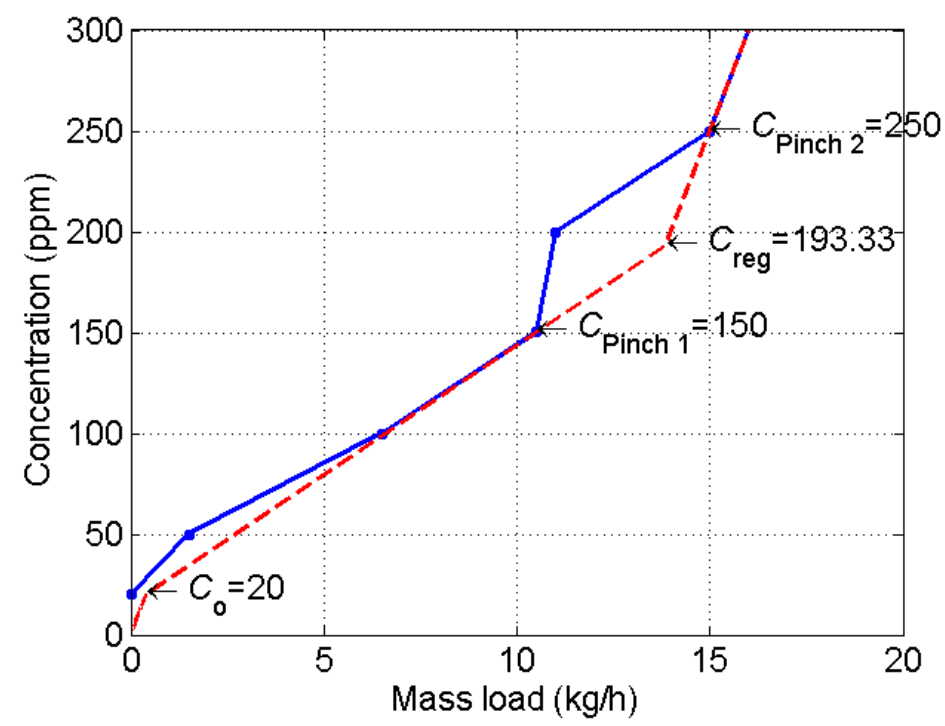

Figure 4. LCC along with WSL for targeting water-regeneration-recycle network with $C_{0}=20 \mathrm{ppm}$ (Example 1) 
The water network design with ZLD is shown in Figure 5. The enhanced nearest neighbour algorithm (NNA)

2 (Shenoy, 2012) is adopted to design the water network. The network constructed is shown as a matching

3 matrix in Figure 5. Note that in the design stage the original limiting data should be considered (rather than

4 the updated ones). The regeneration pinch concentration has been identified at $150 \mathrm{ppm}$ in which the

5 forbidden matches across the pinch point can be determined (shown as shaded cells). Note that the

6 regeneration outlet stream (Regout) is included as a source, while its inlet (Regin) as a sink. The enhanced NNA

7 also determines that process sources SR 3 and SR 4 are purified in regeneration unit before partially recycled

8 to process sinks SK1 and SK2.

\begin{tabular}{|c|c|c|c|c|c|c|c|c|}
\hline & & $F_{\text {Skj }}(\mathrm{t} / \mathrm{h})$ & 50 & 100 & 80 & 57.69 & 70 & 0 \\
\hline & & $C_{\text {Skj }}(p p m)$ & 20 & 50 & 100 & 193.33 & 200 & 0 \\
\hline$F_{\text {SRi }}(t / h)$ & $C_{\text {SRi }}(p p m)$ & $\mathrm{SRi}^{\mathrm{SKj}}$ & SK1 & SK2 & SK3 & Regin & SK4 & WW \\
\hline 20 & 0 & FW & & 18.85 & 1.15 & & & \\
\hline 57.69 & 20 & Regout & 50 & 7.69 & & & & \\
\hline 50 & 50 & SR1 & & 50 & & & & \\
\hline 100 & 100 & SR2 & & 23.46 & 76.54 & & & \\
\hline 70 & 150 & SR3 & & & 2.31 & 32.69 & 35 & 0 \\
\hline 60 & 250 & SR4 & & & & 25 & 35 & 0 \\
\hline
\end{tabular}

Figure 5. Network design for the ZLD system as a matching matrix (Example 1)

The general structure of ACTA is summarised in Figure 6.

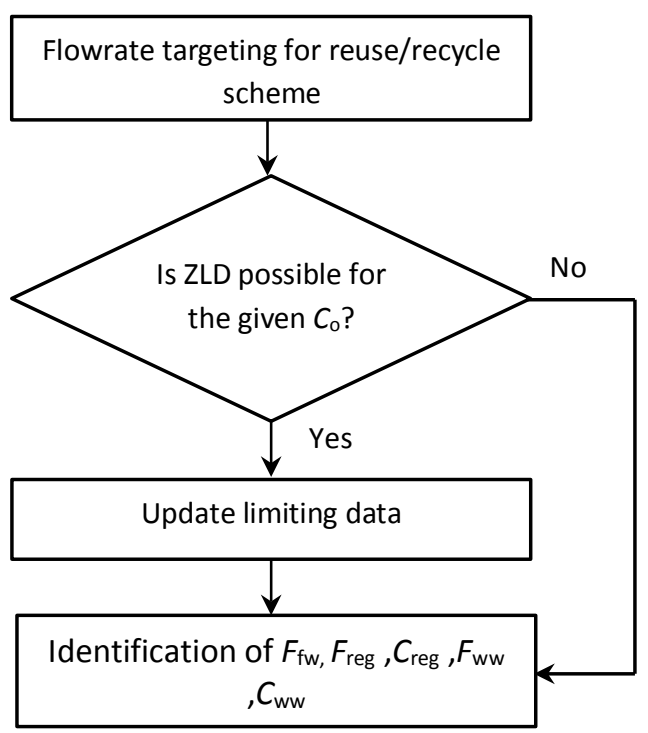

Figure 6. The general structure of ACTA

As mentioned, the use of the improved CTA (step 4) without updating the limiting data may lead to infeasible results. This happens for all $C_{o}$ s that stay between $0 \mathrm{ppm}$ and $C_{o, Z L D}^{\max }$ (i.e. $33.33 \mathrm{ppm}$ ). For the case of $C_{o}=20$ ppm, the original limiting data (Table 1 ) and improved CTA (step 4) determine the $F_{\mathrm{fw}}$, and $F_{\text {reg }}$ as $0 \mathrm{t} / \mathrm{h}$ and 
$81.25 \mathrm{t} / \mathrm{h}$, respectively. Note however that $F_{\mathrm{ww}}$ is calculated as $-20 \mathrm{t} / \mathrm{h}$ with Eq. 4 , which is infeasible. This justifies the necessity of updating the limiting data for ZLD cases with this newly proposed ACTA.

To demonstrate the capability of ACTA, $C_{0}$ of $40 \mathrm{ppm}$ is next used. It is expected that some amount of wastewater will be generated, since this $C_{o}$ is greater than the $C_{o, Z L D}^{\max }$ of $33.33 \mathrm{ppm}$. Following the earlier outlined procedure, the limiting data is updated (as explained in step 3) regardless of $C_{0}$ value because this network has the possibility of achieving ZLD. Next, with the improved CTA (step 4), $F_{\mathrm{fw}}$ and $F_{\text {reg }}$ are identified as $25 \mathrm{t} / \mathrm{h}, 66.67 \mathrm{t} / \mathrm{h}$, respectively. The $C_{\text {reg }}$ is found at $171.25 \mathrm{ppm}$, while the regeneration pinch concentrations are located at $100 \mathrm{ppm}\left(C_{\text {Pinch 1 }}\right)$ and $250 \mathrm{ppm}\left(C_{\text {Pinch 2 }}\right)$, respectively. Next, $F_{\text {ww }}$ is identified as $5 \mathrm{t} / \mathrm{h}$ via Eq. 4, while $C_{w w}$ is found as 250 ppm via Eq. 5. The targeted results are shown using the LCC in Figure 7.

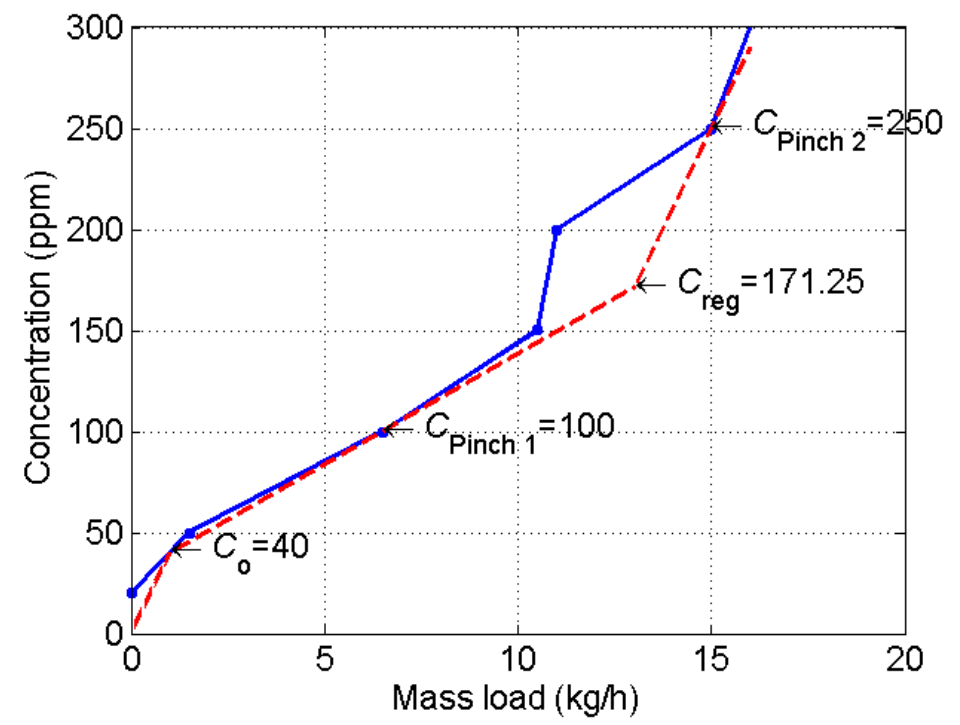

Figure 7. LCC along with WSL for $\mathrm{C}_{0}=40 \mathrm{ppm}$ (Example 1)

Note from Figure 7 that the first regeneration pinch concentration (i.e. $100 \mathrm{ppm}$ ) is different from the earlier case with $C_{0}$ of 20 ppm (150 ppm, see Figure 4). This means that the regeneration pinch points can switch among the turning point of LCC with the change of $C_{0}$. Thus, the assumption made by Agrawal and Shenoy (2006) where the reuse/recycle pinch concentration (i.e. $150 \mathrm{ppm}$ ) is taken as regeneration pinch is not generic enough.

To verify the solution, the enhanced nearest neighbour algorithm (NNA) (Shenoy, 2012) is adopted again to design the water network with $C_{0}=40 \mathrm{ppm}$. The network constructed is shown as a matching matrix in Figure 8. 


\begin{tabular}{|c|c|c|c|c|c|c|c|c|}
\hline & & $F_{\text {SKj }}(\mathrm{t} / \mathrm{h})$ & 50 & 100 & 80 & 66.67 & 70 & 5 \\
\hline & & $C_{\mathrm{SKj}}(\mathrm{ppm})$ & 20 & 50 & 100 & 171.25 & 200 & 250 \\
\hline$F_{\text {SRi }}(t / h)$ & $C_{\mathrm{SRi}}(\mathrm{ppm})$ & $\mathrm{SRi}_{\mathrm{SKj}} \mathrm{SKj}$ & SK1 & SK2 & SK3 & Regin & SK4 & WW \\
\hline 25 & 0 & FW & 25 & & & & & \\
\hline 66.67 & 40 & Regout & 25 & 41.67 & & & & \\
\hline 50 & 50 & SR1 & & 50 & & & & \\
\hline 100 & 100 & SR2 & & 8.33 & 80 & & 11.67 & \\
\hline 70 & 150 & SR3 & & & & 52.5 & 17.5 & \\
\hline 60 & 250 & SR4 & & & & 14.17 & 40.83 & 5 \\
\hline
\end{tabular}

1

Figure 8. Network design presented as matching matrix for Example 1 with $C_{o}=40 \mathrm{ppm}$

As demonstrated, the ACTA is capable of targeting water regeneration-recycle network for different $C_{0} S$ as long as the problem remains feasible. The LCC sets the boundary for a regeneration-recycle system. In other words, for a feasible water network, the WSL should always stay below and touch the LCC at the regeneration pinch point(s). For some special cases where the LCC has the convex turning point below the reuse/recycle pinch point, the WSL may appear above the LCC for the confined range of $C_{0}$. This implies the mass load infeasibility to the problem. Addressing this issue will be the scope of future works. However, since the targeted values from ACTA can be displayed in the graphical form (i.e. LCC), one can cross check the mass load feasibility of the results.

\section{Example 2- Fixed load problem}

The capability of ACTA in handling FL problems is next demonstrated with Example 2 taken from Feng et al. (2007), with the limiting data given in Table 5. The targeted values for reuse/recycle network can be readily determined by employing the original CTA (Agrawal and Shenoy, 2006) as outlined in step 1. The detailed procedure is omitted for brevity. The freshwater and wastewater flowrates are both determined as $105 \mathrm{t} / \mathrm{h}$, with $\mathrm{C}_{\mathrm{pr}}$ located at $100 \mathrm{ppm}$.

Table 5. Limiting data for example 2

\begin{tabular}{llllll}
\hline SKj & $F_{\text {SKj }}(\mathrm{t} / \mathrm{h})$ & $C_{\text {SKj }}(\mathrm{ppm})$ & $\mathrm{SRi}$ & $F_{\mathrm{SRi}}(\mathrm{t} / \mathrm{h})$ & $C_{\mathrm{SRi}}(\mathrm{ppm})$ \\
\hline 1 & 60 & 0 & 1 & 60 & 100 \\
2 & 40 & 50 & 2 & 40 & 150 \\
3 & 100 & 75 & 3 & 100 & 100 \\
4 & 50 & 100 & 4 & 50 & 125 \\
\hline
\end{tabular}

The ACTA next determines that the water network does not exhibit ZLD possibility as it does not encounter any water loss. Thus, the limiting data need not be updated and the improved CTA (Table 6) can be implemented with the use of original limiting data. It is assumed that a regeneration unit with $C_{0}$ of $40 \mathrm{ppm}$ is used. The ACTA procedure determines that the $F_{\mathrm{fw}}$, and $F_{\text {reg }}$ are identified as $60 \mathrm{t} / \mathrm{h}$ and $75 \mathrm{t} / \mathrm{h}$, respectively; while $C_{\text {reg }}$ is found as 110 ppm. Next, regeneration pinch concentrations are located at 100 and 125 ppm, respectively. . 
2 results are completely in agreement with those reported in Feng et al. (2007).

Table 6. Determination of $F_{\mathrm{fw}}, F_{\mathrm{reg}}$, and $C_{\mathrm{reg}}$ with improved CTA for $C_{\mathrm{o}}=40 \mathrm{ppm}$ (Example 2)

\begin{tabular}{llllllll}
\hline$k$ & $C_{\mathrm{k}}(\mathrm{ppm})$ & Net. $F_{\mathrm{k}}(\mathrm{t} / \mathrm{h})$ & $\Delta m_{\mathrm{k}}(\mathrm{kg} / \mathrm{h})$ & Cum. $\Delta m_{\mathrm{k}}(\mathrm{kg} / \mathrm{h})$ & $F_{\text {fw }, \mathrm{k}}(\mathrm{t} / \mathrm{h})$ & $F_{\text {reg }, \mathrm{k}}(\mathrm{t} / \mathrm{h})$ & $C_{\text {reg, } \mathrm{k}}(\mathrm{ppm})$ \\
\hline 1 & 0 & 60 & 2.4 & 0 & 0 & & \\
2 & $C_{0}=40$ & 60 & 0.6 & 2.4 & $F_{\mathrm{fw}}=60$ & & \\
3 & 50 & 100 & 2.5 & 3 & 60 & 0 & \\
4 & 75 & 200 & 5 & 5.5 & 73.33 & 28.57 & \\
5 & $C_{\text {pinch } 1}=100$ & 90 & 2.25 & 10.5 & 105 & $F_{\text {reg }}=75$ & 100 \\
6 & $C_{\text {pinch } 2}=125$ & 40 & 1 & 12.75 & 102 & & $C_{\text {reg }}=110$ \\
7 & 150 & 0 & $(0)$ & 13.75 & 91.67 & & 103.33 \\
8 & $(155)$ & & & 13.75 & 88.71 & & 93.33 \\
\hline
\end{tabular}

4

5 The network design for this case using enhanced NNA (Shenoy, 2012) is shown as a matching matrix in Figure

6 9. The Local Recycle (LR) matches are identified and eliminated to simplify the network structure. Thus the

7 limiting data need to be modified accordingly.

\begin{tabular}{|c|c|c|c|c|c|c|c|c|}
\hline & & $F_{\mathrm{SKj}}(\mathrm{t} / \mathrm{h})$ & 60 & 40 & 10041.67 & 50 & 75 & 60 \\
\hline & & $C_{\text {SKj }}(p p m)$ & 0 & 50 & 7540 & 100 & 110 & 141.67 \\
\hline$F_{\text {SRi }}(t / h)$ & $C_{\text {SRi }}(\mathrm{ppm})$ & $\mathrm{SRi}_{\mathrm{SR} i}^{\mathrm{SKj}}$ & SK1 & SK2 & SK3 & SK4 & Regin & WW \\
\hline 60 & 0 & FW & 60 & & & & & \\
\hline 75 & 40 & Regout & & 33.33 & 41.67 & & & \\
\hline 60 & 100 & SR1 & & 6.67 & & 50 & 3.33 & \\
\hline 10041.67 & 100 & SR3 & & & 58.33 LR & & 41.67 & \\
\hline 50 & 125 & SR4 & & & & & 30 & 20 \\
\hline 40 & 150 & SR2 & & & & & & 40 \\
\hline
\end{tabular}

8

9 Figure 9. Network design presented as matching matrix for Example 2 with $C_{\mathrm{o}}=40 \mathrm{ppm}$

\section{Conclusions}

A new targeting procedure based on Pinch Analysis named as Automated Composite Table Algorithm (ACTA) has been developed in this work, for the targeting of water-regeneration-recycle network. This targeting technique is the enhanced version of Composite Table Algorithm (CTA) by taking into consideration the possibility of the zero liquid discharge (ZLD). The ACTA generates the updated limiting data and identify the key parameters automatically. In the conventional CTA targeting procedure, the reuse/recycle pinch point was taken as the regeneration pinch point for the water regeneration-recycle network. However, as demonstrated 
in this study, the regeneration pinch point may vary with different post-regeneration concentration. Thus, the traditional CTA may produce unreliable network targets for some cases. This limitation is overcome by the ACTA. The latter can handle various types of problems in water regeneration-recycle system, including both ZLD and non-ZLD networks, for both FL and/or FF problems. Typical FF and FL literature problems have been used to demonstrate the applicability of the ACTA. In future work, the ACTA procedure can be extended to determine the optimum $C_{0}$ values automatically, multi-contaminant cases, as well as to handle partitioning regeneration units with significant water losses.

\section{Acknowledgment}

The University of Nottingham Malaysia Campus Matched Fund on the support of this research work is gratefully acknowledged.

\section{Appendix - Numerical procedure for step 2 of the ACTA}

The aim of this appendix is to present the detailed algebraic procedure for the step 2 of the ACTA which evaluates the condition for ZLD possibility. Example 1 is given for explanation. The numerical procedure is outlined as follows:

\section{Identification of line $A B$ formula}

For line $A B$ in Figure 3, the coordinates of point $A$ and point $B$ are determined using Eq. A1. Assuming that pure freshwater is used, the $C_{\mathrm{fw}}$ value is set to zero. In addition, cumulative mass loads (Cum. $\Delta m_{\mathrm{k}}$ ) correspond to the first concentration level is also zero (Table 2). Thus, Point A is located at the origin in Figure 3. On the other hand, the coordinates of point $B$ are related to the amount of water loss and reuse/recycle pinch concentration (i.e. $C_{p r}=150$ ppm). The last entry in the column 3 of Table $2\left(\mathrm{NetF}_{\mathrm{k}-1}\right.$ where $\left.\mathrm{k}=\max (\mathrm{k})\right)$ identifies the amount of water loss (i.e. $20 \mathrm{t} / \mathrm{h}$ ).

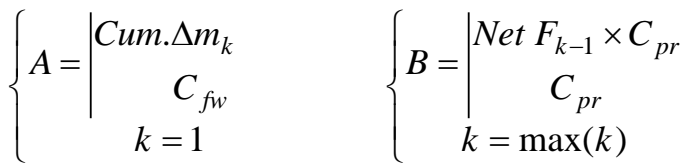

\section{Construction of LCC segment formula}

Having introduced line GH as an LCC segment below the reuse/recycle pinch point (see Figure 3), the coordinates of points $\mathrm{G}$ and $\mathrm{H}$ are identified using Eq. A2. For example 1, three segments are identified. Segments 1 has a starting point of $G(0,20)$ and ending of $H(1.550)$; segment 2 has the starting point of $G(1.5$, $50)$ and the ending point of $H(6.5100)$ and segment 3 has the starting point of $G(6.5,100)$ and ending point of $H(10.5,150)$.

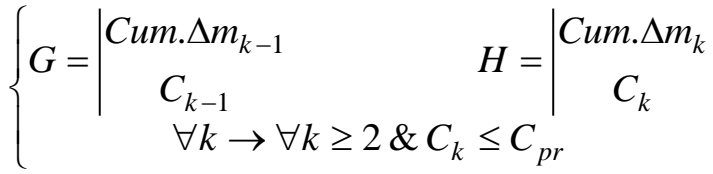



between any of the $\mathrm{G}$ and $\mathrm{H}$ points given above to be able the forming of the pseudo segment (introduced in step 2 of the ACTA procedure). For example 1, the intersection point is found at the coordinates of (0.66, 33.33), which lies between $\mathrm{G}$ and $\mathrm{H}$ points of segment 1. The $\mathrm{Y}$ coordinate of intersection point is also taken as $C_{o, Z L D}^{\max }$ (i.e. $C_{o, Z L D}^{\max }=33.33 \mathrm{ppm}$ ). The $C_{o, Z L D}^{\max }$ value is essential parameter to update the limiting data in step 3 of the ACTA.

\section{References}

Agrawal V, Shenoy, UV (2006) Unified conceptual approach to targeting and design of water and hydrogen networks. AIChE J 52:1071-1082

Aly S, Abeer S, Awad M (2005) A new systematic approach for water network design. Clean Technol Environ Policy 7(3):154-161

Bandyopadhyay S, Ghanekar MD, Pillai HK (2006) Process water management. Ind Eng Chem Res 45(15):52875297

Deng C, Feng X, Bai J (2008) Graphically based analysis of water system with zero liquid discharge. Chem Eng Res Des 86:165-171

Deng C, Feng X (2011) Targeting for conventional and property-based water network with multiple resources. Ind Eng Chem Res 50(7):3722-3737

Deng C, Feng X, Wen Z (2013) Optimization of water network integrated with process models. Clean Technol Environ Policy 15(3):473-487

Deng C, Shi C, Feng X, Foo DCY (2016) Flowrate targeting for concentration-and property-based total water network with multiple partitioning interception units. Ind Eng Chem Res DOI: 10.1021 /acs .iecr. 5 b03203 El-Halwagi MM (2006) Process integration. Amsterdam: Academic

Feng X, Bai J, Zheng X (2007) On the use of graphical method to determine the targets of single-contaminant regeneration recycling water systems. Chem Eng Sci 62:2127-2138

Foo DCY, Manan ZA, El-Halwagi MM (2006) Correct identification of limiting water data for water network synthesis. Clean Technol Environ Policy 8(2): 96-104

Foo DCY (2009) State-of-the-art review of pinch analysis techniques for water network synthesis. Ind Eng Chem Res 48:5125-5159

Foo DCY (2008) Flowrate targeting for threshold problems and plant-wide integration for water network synthesis. J Environ Manage 88:253-274

Foo DCY (2012) Process integration for resource conservation CRC Press: Boca Raton, FL, U.S Institute of Chemical Engineers (IChemE) (2014) Chemical Engineering Matters. IChemE's technical strategy scope, applications and implications. 2nd Edition. Rugby, UK

Hallale NA (2002) new graphical targeting method for water minimisation. Adv Environ Res 6(3):377-390.

Klemeš JJ (2013) Handbook of process integration (PI): minimisation of energy and water use, waste and emissions. Elsevier

Klemeš JJ, Varbanov PS, Alwi SRWW, Manan ZA (2014) Process integration and intensification: saving energy, water and resources. Walter de Gruyter GmbH \& Co KG: Berlin, Germany

Kuo WCJ, Smith R (1998) Design of water-using systems involving regeneration. Proc Saf Envr Prot 76:94-114 Manan ZA, Tan YL, Foo DCY (2004) Targeting the minimum water flow rate using water cascade analysis technique. AIChE J 50(12):3169-3183

Ng DKS, Foo DCY, Tan RR, Tan YL (2007) Ultimate flowrate targeting with regeneration placement. Chem Eng Res Des 85(9):1253-1267

Parand R, Yao HM, Tadé MO, Pareek V (2013a) Composite table algorithm - A powerful hybrid pinch targeting method for various problems in water integration. Int J Chem Eng Appl 4(4):224-228

Parand R, Yao HM, Tadé MO, Pareek V (2013b) Targeting water utilities for the threshold problem without waste discharge. Chem Eng Res Des 91(12):2569-2578

Parand R, Yao, HM, Pareek V, Tadé, MO (2014) Use of pinch concept to optimize the total water regeneration network. Ind Eng Chem Res 53:3222-3235

Polley G, Polley HL (2000) Design better water networks. Chem Eng Prog 96:47-52 
Saw SY, Lee L, Lim MH, Foo DCY, Chew IML, Tan RR, Klemeš J (2011) An extended graphical targeting technique for direct reuse/recycle in concentration- and property-based resource conservation networks. Clean Technol Environ Policy 13(2):347-357

Smith R (2016) Chemical process design and integration, 2nd Edition: Wiley New York

Steffen W, Richardson K, Rockström J, Cornell SE, Fetzer I, Bennett EM, Biggs R, Carpenter SR, de Vries W, de Wit CA, Folke C, Gerten D, Heinke J, Mace GM, Persson LM, Ramanathan V, Reyers B, Sorlin S (2015) Planetary boundaries: Guiding Human development on a changing planet. Science 347 no. 6223

Shenoy UV (2012) Enhanced nearest neighbors algorithm for design of water networks. Chem Eng Sci 84:197206

10 Takama N, Kuriyama T, Shiroko K, Umeda T (1980) Optimal water allocation in a petroleum refinery. Comput. 11 Chem Eng 4:251-258

12 Wang YP, Smith R (1994) Wastewater minimisation. Chem Eng Sci 49:981-1006 